\title{
Octonionic Gravi-electromagnetism and Dark Matter
}

\author{
B. C. Chanyal, V. K. Sharma and O. P. S. Negi \\ Department of Physics \\ Kumaun University, S. S. J. Campus Almora-263601 (U.K.) India \\ Email:- bcchanyal@gmail.com \\ vishalphysics1985@gmail.com \\ ops_negi@yahoo.co.in
}

\begin{abstract}
An attempt has been made to analyse the the role of octonions in various unified field theories associated with dyons and the dark matter. Starting with the split octonion algebra and its properties, we have discussed the octonionic unified gauge formulation for $S U(2) \times U(1)$ electroweak theory and $S U(3) \times S U(2) \times U(1)$ grand unified theory. Describing the octonion eight dimensional space as the combination of two quaternionic spaces (namely associated with the electromagnetic interaction (EM-space) and linear gravitational interaction (G-space)), we have reexamined the unified picture of EM-G space in terms of octonionic split formulation in consistent manner. Consequently, we have obtained the various field equations for unified gravi-electromagnetic interactions. Furthermore, we have reconstructed the field equations of hot and cold dark matter in terms of split octonions. It is shown that the difference between the octonion cold dark matter (OCDM) and the octonion hot dark matter (OHDM) is significant in the formulating of structure of these two, because the velocities of octonion hot dark matter cause it to wipe out structure on small scales.
\end{abstract}

Key Words: octonions, split-octonions, monopole, dyons, non-Abelian gauge theories, octonion dark matter.

\section{Introduction}

According to celebrated Hurwitz theorem there exists [1] a set of four division algebra $\{\mathbb{R}, \mathbb{C}, \mathbb{H}, \mathcal{O}\}$ namely the real numbers $(\mathbb{R})$, complex numbers $(\mathbb{C})$, quaternions $(\mathbb{H})[2,3]$ and octonions $(\mathcal{O})$ 4, 5, 6. All these four algebra's are alternative with totally anti symmetric associators. Real number explains well the classical Newtonian mechanics while the complex number plays an important role for the explanation beyond the framework of quantum theory and relativity. Real and complex numbers are limited only up to two dimensions while quaternions are extended to four dimensions (one real and three imaginaries), on the other hand the octonions represent eight dimensions (one real and seven imaginaries). Real and complex numbers are commutative and 
associative. Quaternions are associative but not commutative while the octonions are neither commutative and nor associative but alternative. Quaternions are well connected [7] with the familiar $S U(2)$ group and naturally unify [8, 9] electromagnetism and weak force responsible for the electroweak $S U(2) \times U(1)$ sector of standard model. The octonion analysis has played an important role in the context of various physical problems [10 -17] higher dimensional supersymmetry, super gravity and super strings. Octonions are also [13 -15] used for unification program of strong interaction with successful gauge theory of fundamental interactions. A theoretical description of the leptons and quark structure of hadrons has already been proposed by Günaydin and Gursey [18] in the context of octonionic quantum mechanics in terms of octonionic Hilbert space.

Rather, there has been continuing interests of many authors [19 -21] towards the developments of wave equation and octonion form of Maxwell's equations. We [22 -31] have also discussed the significance of octonions to develop generalized octonion electrodynamics, generalized split octonion electrodynamics, octonion quantum chromodynamics, octonionic non-Abelian gauge theory, octonion and conservation laws for dyons, octonion electrodynamics in isotropic and chiral medium, octonion massive electrodynamics, octonionic representation of superstring theory, octonion model of dark matter, octonion symmetric Dirac-Maxwell's equations and obtained the corresponding field equations and equation of motion in compact and consistence way. Keeping in mind the recent update of theoretical physics, we have made to analyze the the role of octonions in various unified field theories associated with dyons and the dark matter. Starting with the split octonion algebra and its properties, we have discussed the octonionic unified gauge formulation for $S U(2) \times U(1)$ electroweak theory and $S U(3) \times S U(2) \times U(1)$ grand unified theory. Describing the octonion eight dimensional space as the combination of two quaternionic spaces (namely associated with the electromagnetic interaction (EM-space) and linear gravitational interaction (G-space)), we have reexamined the unified picture of EM-G space in terms of octonionic split formulation in consistent manner. Consequently, we have obtained the various field equations for unified gravi-electromagnetic interactions. Furthermore, we have reconstructed the field equations of hot and cold dark matter in terms of split octonions. It is shown that the difference between the octonion cold dark matter (OCDM) and the octonion hot dark matter (OHDM) is significant in the formulating of structure of these two, because the velocities of octonion hot dark matter cause it to wipe out structure on small scales. As such, there exists more cold dark matter than that of hot dark matter in nature supported by octonionic field equations.

\section{The Split Octonion}

An octonion $\mathcal{O}$ is expressed [22-29] as a real linear combination of the unit octonions $\left(e_{0}, e_{1}, e_{2}, e_{3}\right.$, $\left.e_{4}, e_{5}, e_{6}, e_{7}\right)$ as

$$
\mathcal{O} \simeq\left(\mathcal{O}_{0}, \mathcal{O}_{1}, \mathcal{O}_{2}, \mathcal{O}_{3}, \mathcal{O}_{4}, \mathcal{O}_{5}, \mathcal{O}_{6}, \mathcal{O}_{7}\right)=\mathcal{O}_{0} e_{0}+\sum_{A=1}^{7} \mathcal{O}_{A} e_{A}
$$

where $e_{A}(A=1,2, \ldots ., 7)$ are imaginary octonion units and $e_{0}$ is the real octonion unit element. The octet $\left(e_{0}, e_{1}, e_{2}, e_{3}, e_{4}, e_{5}, e_{6}, e_{7}\right)$ is known as the octonion basis where $e_{A}$ satisfy the following 
multiplication rules

$$
\begin{aligned}
e_{0} & =1, \quad e_{A}^{2}=-1, \quad e_{0} e_{A}=e_{A} e_{0}=e_{A}, \\
e_{A} e_{B} & =-\delta_{A B} e_{0}+f_{A B C} e_{C} \cdot(A, B, C=1,2, \ldots \ldots .7)
\end{aligned}
$$

The structure constants $f_{A B C}$ are completely antisymmetric and take the value 1, i.e.

$$
f_{A B C}=+1 \longmapsto(A B C) \equiv(123),(471),(257),(165),(624),(543),(736)
$$

Therefore, we get the following relations among octonion basis elements as

$$
\left[e_{A}, e_{B}\right]=2 f_{A B C} e_{C},\left\{e_{A}, e_{B}\right\}=-\delta_{A B} e_{0}, e_{A}\left(e_{B} e_{C}\right) \neq\left(e_{A} e_{B}\right) e_{C}
$$

where $\delta_{A B}$ is the usual Kronecker delta-Dirac symbol.

Like wise, the split octonions [25, 26] are a non associative extension of split quaternions (associative). Split octonions differ from the octonions in the signature of quadratic form. The split octonion have a signature $(4,4)$ whereas the octonions have positive signature $(8,0)$. The Cayley algebra of octonion over the field of complex number $\mathbb{C}_{\mathbb{C}}=\mathbb{C} \otimes C$ is visualized as the algebra of split octonions with its following basis elements,

$$
\left\{\begin{array}{lll}
u_{0}=\frac{1}{2}\left(e_{0}+i e_{7}\right) ; & u_{0}^{*}=\frac{1}{2}\left(e_{0}-i e_{7}\right) ; & \\
u_{j}=\frac{1}{2}\left(e_{j}+i e_{j+3}\right) ; & u_{j}^{*}=\frac{1}{2}\left(e_{j}-i e_{j+3}\right) ; \quad(\forall j=1,2,3)
\end{array}\right.
$$

where $i=\sqrt{-1}$ is assumed to commute with $e_{A}(A=1,2, \ldots \ldots, 7)$ octonion units. The split octonion basis elements $\left(u_{0}, u_{j}, u_{0}^{*}, u_{j}^{*}\right)$ satisfy the following multiplication rules,

$$
\begin{aligned}
& u_{i} u_{j}=\epsilon_{i j k} u_{k}^{*} ; \quad u_{i}^{*} u_{j}^{*}=-\epsilon_{i j k} u_{k}^{*} ; \quad(\forall i, j, k=1,2,3) \\
& u_{i} u_{j}^{*}=-\delta_{i j} u_{0} ; \quad u_{i} u_{0}=0 ; \quad u_{i}^{*} u_{0}=u_{i}^{*} ; \\
& u_{i}^{*} u_{j}=-\delta_{i j} u_{0} ; \quad u_{i} u_{0}^{*}=u_{i} ; \quad u_{i}^{*} u_{0}^{*}=0 ; \\
& u_{0} u_{i}=u_{i} ; \quad u_{0}^{*} u_{i}=0 ; \quad u_{0} u_{i}^{*}=0 ; \\
& u_{0}^{*} u_{i}^{*}=u_{i} ; \quad u_{0}^{2}=u_{0} ; \quad u_{0}^{* 2}=u_{0}^{*} ; \quad u_{0} u_{0}^{*}=u_{0}^{*} u_{0}=0 .
\end{aligned}
$$

Thus the multiplication table of split octonionic basis elements is given in Table-1.

\begin{tabular}{ccccccccc}
\hline$\cdot$ & $u_{0}^{*}$ & $u_{1}^{*}$ & $u_{2}^{*}$ & $u_{3}^{*}$ & $u_{0}$ & $u_{1}$ & $u_{2}$ & $u_{3}$ \\
$u_{0}^{*}$ & $u_{0}^{*}$ & $u_{1}^{*}$ & $u_{2}^{*}$ & $u_{3}^{*}$ & 0 & 0 & 0 & 0 \\
$u_{1}^{*}$ & 0 & 0 & $u_{3}$ & $-u_{2}$ & $u_{1}^{*}$ & $-u_{0}^{*}$ & 0 & 0 \\
$u_{2}^{*}$ & 0 & $-u_{3}$ & 0 & $u_{1}$ & $u_{2}^{*}$ & 0 & $-u_{0}^{*}$ & 0 \\
$u_{3}^{*}$ & 0 & $u_{2}$ & $-u_{1}$ & 0 & $u_{3}^{*}$ & 0 & 0 & $-u_{0}^{*}$ \\
$u_{0}$ & 0 & 0 & 0 & 0 & $u_{0}$ & $u_{1}$ & $u_{2}$ & $u_{3}$ \\
$u_{1}$ & $u_{1}$ & $-u_{0}$ & 0 & 0 & 0 & 0 & $u_{3}^{*}$ & $-u_{2}^{*}$ \\
$u_{2}$ & $u_{2}$ & 0 & $-u_{0}$ & 0 & 0 & $-u_{3}^{*}$ & 0 & $u_{1}^{*}$ \\
$u_{3}$ & $u_{3}$ & 0 & 0 & $-u_{0}$ & 0 & $u_{2}^{*}$ & $-u_{1}^{*}$ & 0 \\
\hline
\end{tabular}

Table 1: Split-Octonion Multiplication Table 
The automorphism group of octonion is described [32, 33] as $G_{2}$. Its subgroup which leaves imaginary octonion unit $e_{7}$ invariant (or equivalently the idempotents $u_{0}$ and $u_{0}^{*}$ ) is $S U(3)$. Here the units $u_{j}$ and $u_{j}^{*}(j=1,2,3)$ transform respectively like a triplet and anti triplet respectively associated with colour and anti colour triplets of $S U(3)$ group. We may now introduce a convenient realization for the basis elements $\left(u_{0}, u_{j}, u_{0}^{*}, u_{j}^{*}\right)$ in term of Pauli's spin matrices as

$$
\begin{cases}u_{0}=\left(\begin{array}{cc}
0 & 0 \\
0 & 1
\end{array}\right) ; & u_{0}^{*}=\left(\begin{array}{cc}
1 & 0 \\
0 & 0
\end{array}\right) ; \\
u_{j}=\left(\begin{array}{cc}
0 & 0 \\
e_{j} & 0
\end{array}\right) ; & u_{j}^{*}=\left(\begin{array}{cc}
0 & -e_{j} \\
0 & 0
\end{array}\right) . \quad(\forall j=1,2,3)\end{cases}
$$

So, the split octonion algebra is now expressed in terms of $2 \times 2$ Zorn's vector matrices components of which are scalar and vector parts of a quaternion, i.e.

$$
\mathcal{O}=\left\{\left(\begin{array}{cc}
m & \vec{p} \\
\vec{q} & n
\end{array}\right) ; \quad m, n \in S c(\mathbb{H}) ; \quad \& \vec{p}, \vec{q} \in V e c(\mathbb{H})\right\}
$$

As such, we write an arbitrary split octonion $A \in \mathcal{O}$ in terms of following $2 \times 2$ Zorn's vector matrix realization as

$$
A=a u_{0}^{*}+b u_{0}+x_{i} u_{i}^{*}+y_{i} u_{i} \simeq\left(\begin{array}{cc}
a & -\vec{x} \\
\vec{y} & b
\end{array}\right),
$$

where $a$ and $b$ are scalars and $\vec{x}$ and $\vec{y}$ are three vectors. Thus the product of two octonions in terms of following $2 \times 2$ Zorn's vector matrix realization is expressed as

$$
\left(\begin{array}{cc}
a & \vec{x} \\
\vec{y} & b
\end{array}\right)\left(\begin{array}{cc}
c & \vec{u} \\
\vec{v} & d
\end{array}\right)=\left(\begin{array}{cc}
a c+(\vec{x} \cdot \vec{v}) & a \vec{u}+d \vec{x}+(\vec{y} \times \vec{v}) \\
c \vec{y}+b \vec{v}-(\vec{x} \times \vec{u}) & b d+(\vec{y} \cdot \vec{u})
\end{array}\right)
$$

where $(\times)$ denotes the usual vector product, $e_{j}(j=1,2,3)$ with $e_{j} \times e_{k}=\epsilon_{j k l} e_{l}$ and $e_{j} e_{k}=-\delta_{j k}$. Octonion conjugate of equation (9) in terms of $2 \times 2$ Zorn's vector matrix realization is now defined as

$$
\bar{A}=a u_{0}+b u_{0}^{\star}-x_{i} u_{i}^{\star}-y_{i} u_{i} \simeq\left(\begin{array}{cc}
b & \vec{x} \\
-\vec{y} & a
\end{array}\right) .
$$

The norm of $A$ is defined as

$$
N(A)=\bar{A} A=A \bar{A}=(a b+\vec{x} \cdot \vec{y}) \hat{1}=n(A) \hat{1}
$$

where $\hat{1}$ is the identity elements of matrix order $2 \times 2$, and the expression $n(A)=(a b+\vec{x} \cdot \vec{y})$ defines the quadratic form which admits the composition as

$$
n(\vec{A} \cdot \vec{B})=n(\vec{A}) n(\vec{B}), \quad(\forall \vec{A}, \vec{B} \in \mathcal{O})
$$

Thus, we may easily express the Euclidean or Minkowski four vector in split octonion formulation 
in terms of $2 \times 2$ Zorn's vector matrix realizations. Consequently, any four-vector $A_{\mu}$ (complex or real) can equivalently be written in terms of the following Zorn matrix realization as

$$
\mathbb{Z}(A)=\left(\begin{array}{cc}
x_{4} & -\vec{x} \\
\vec{y} & y_{4}
\end{array}\right) ; \quad \mathbb{Z}(\bar{A})=\left(\begin{array}{cc}
x_{4} & \vec{x} \\
-\vec{y} & y_{4}
\end{array}\right) .
$$

The split octonion valued space time vector $\mathbb{Z}^{\mu}(\mu=0,1,2,3)$ in terms of the $4 \times 4$ (space time vector-valued) Zorn matrix $\mathbb{Z}_{a b}^{\mu}$ may now be expressed as [10],

$$
\mathbb{Z}^{\mu}=x_{0}^{\mu} u_{0}^{*}+y_{0}^{\mu} u_{0}+x_{j}^{\mu} u_{j}^{*}+y_{j}^{\mu} u_{j} \simeq\left(\begin{array}{cc}
x_{0}^{\mu} e_{0} & -x_{j}^{\mu} e_{j} \\
y_{j}^{\mu} e_{j} & y_{0}^{\mu} e_{0}
\end{array}\right), \quad(\forall j=1,2,3)
$$

where $\mu(\forall 0,1,2,3)$ represents the internal four dimensional space. Here $\mu=0$ describes $U(1)$ abelian gauge structure while $\mu=j(\forall j=1,2,3)$ is associated with the $S U(2)$ non-Abelian gauge structure [26]. Here $x_{0}^{\mu}, x_{j}^{\mu}, y_{0}^{\mu}, y_{j}^{\mu}$ are real valued variables for abelian and non-Abelian gauge fields. When the space time metric [10, 11] is $\eta_{\mu \nu} \hat{1}_{4 \times 4}$, the bi linear term

$$
\begin{aligned}
\frac{1}{4} \operatorname{Trace}\left[\eta_{\mu \nu} \mathbb{Z}^{\mu} \cdot \mathbb{Z}^{\nu}\right] & =\frac{1}{4} \eta_{\mu \nu}\left[x_{0}^{\mu} x_{0}^{\nu}+y_{0}^{\mu} y_{0}^{\nu}+x_{j}^{\mu} x_{j}^{\nu}+y_{j}^{\mu} y_{j}^{\nu}\right] \operatorname{Trace}\left[\hat{1}_{2 \times 2}\right] \\
& =\frac{1}{2} \eta_{\mu \nu}\left[x_{0}^{\mu} x_{0}^{\nu}+y_{0}^{\mu} y_{0}^{\nu}+x_{j}^{\mu} x_{j}^{\nu}+y_{j}^{\mu} y_{j}^{\nu}\right]
\end{aligned}
$$

describes the inner product. The octonion conjugation is accordingly defined as

$$
\overline{\mathbb{Z}}^{\mu}=x_{0}^{\mu} u_{0}+y_{0}^{\mu} u_{0}^{*}-x_{j}^{\mu} u_{j}^{*}-y_{j}^{\mu} u_{j} \simeq\left(\begin{array}{cc}
y_{0}^{\mu} e_{0} & x_{j}^{\mu} e_{j} \\
-y_{j}^{\mu} e_{j} & x_{0}^{\mu} e_{0}
\end{array}\right),
$$

while the Hermitian conjugation is described [10, 11] as

$$
\left(\mathbb{Z}^{\mu}\right)^{\dagger}=\left(x_{0}^{\mu}\right)^{*} u_{0}+\left(y_{0}^{\mu}\right)^{*} u_{0}^{*}-\left(x_{j}^{\mu}\right)^{*} u_{j}^{*}-\left(y_{j}^{\mu}\right)^{*} u_{j} \simeq\left(\begin{array}{cc}
\left(y_{0}^{\mu}\right)^{*} e_{0} & \left(x_{j}^{\mu}\right)^{*} e_{j} \\
-\left(y_{j}^{\mu}\right)^{*} e_{j} & \left(x_{0}^{\mu}\right)^{*} e_{0}
\end{array}\right) .
$$

The Zorn matrix product of two split octonions

$$
\mathcal{A}=\left(\begin{array}{cc}
A_{0} e_{0} & -A_{i} e_{i} \\
B_{i} e_{i} & B_{0} e_{0}
\end{array}\right) \quad \text { and } \quad \mathcal{B}=\left(\begin{array}{cc}
C_{0} e_{0} & -C_{i} e_{i} \\
D_{i} e_{i} & D_{0} e_{0}
\end{array}\right)
$$

is defined by

$$
\mathcal{A} \mathcal{B}=\left(\begin{array}{cc}
\left(A_{0} C_{0}+A_{j} D_{j}\right) e_{0} & -\left(A_{0} C_{k}+D_{0} A_{k}+\epsilon_{i j k} B_{i} D_{j}\right) e_{k} \\
\left(C_{0} B_{k}+B_{0} D_{k}+\epsilon_{i j k} A_{i} C_{j}\right) e_{k} & \left(B_{0} D_{0}+B_{i} C_{i}\right) e_{0}
\end{array}\right) .
$$

Accordingly, the octonion $A^{\mu}(x)$ with a space-time index [34, may now be written in terms of the split generators as

$$
A^{\mu}(x)=a^{\mu}(x) u_{0}^{*}+b^{\mu}(x) u_{0}+X_{i}^{\mu}(x) u_{i}^{*}+Y_{i}^{\mu}(x) u_{i}, \quad(i=1,2,3)
$$

where the coefficients of $A^{\mu}(x)$ transform like vectors under space-time transformations. As such, 
the space-time covariant derivative of $A^{\mu}(x)$ can be written as the following form:

$$
\begin{aligned}
& A_{+; \alpha}^{\mu}=A_{, \alpha}^{\mu}+\Omega_{\rho \alpha}^{\mu} A^{\rho}, \\
& A_{-; \alpha}^{\mu}=A_{, \alpha}^{\mu}+\Omega_{\alpha \rho}^{\mu} A^{\rho},
\end{aligned}
$$

where $\Omega_{\rho \alpha}^{\mu} \rightarrow \Omega_{\rho \alpha}^{\mu} \cdot \mathbf{1}_{2 \times 2}$ is the affinity of the nonsymmetric theory [34] and $\mathbf{1}_{2 \times 2} \simeq\left(u_{0}^{*}+u_{0}\right)$ is the unit element of the split octonion algebra. Similarly the space-time curvature is given by $R_{\mu \nu \rho}^{\sigma} . \mathbf{1}_{2 \times 2}$, where $R_{\mu \nu \rho}^{\sigma}$ is the curvature of nonsymmetric theory.

\section{Octonionic electroweak formulation}

In order to discuss the role of split octonion in particle physics, we describe the $U(1) \times S U(2)$ electroweak gauge formulation in terms of the split octonion representation. Thus, we may write an octonion as the combination of two gauge fields (i.e. $\mathfrak{A}_{\mu}$ and $\mathfrak{B}_{\mu}$ are respectively associated with electric and magnetic charges) expanded in terms of quaternions, i.e.

$$
\left(\begin{array}{c}
\mathfrak{A}_{\mu} \\
\mathfrak{B}_{\mu}
\end{array}\right) \longmapsto\left(\begin{array}{c}
\mathfrak{A}_{\mu}^{0} e_{0}+\mathfrak{A}_{\mu}^{a} e_{a} \\
\mathfrak{B}_{\mu}^{0} e_{0}+\mathfrak{B}_{\mu}^{a} e_{a}
\end{array}\right), \quad(\forall a=1,2,3)
$$

where the components $\mathfrak{A}_{\mu}^{0}$ and $\mathfrak{B}_{\mu}^{0}$ are described respectively as the electric and magnetic four potentials of dyons for $U(1)$ gauge formalism. Rather, $\mathfrak{A}_{\mu}^{a}$ and $\mathfrak{B}_{\mu}^{a}$ represent two non-Abelian $S U(2)$ gauge field due to the presence of electric and magnetic charges of dyons. As such, the covariant derivative for the case of double fold degeneracy of $U(1) \times S U(2)$ gauge theory is defined as

$$
\mathscr{D}_{\mu}=\left(\begin{array}{cc}
\mathscr{D}_{\mu}^{\mathrm{e}} & 0 \\
0 & \mathscr{D}_{\mu}^{\mathrm{g}}
\end{array}\right) \simeq\left(\begin{array}{cc}
\partial_{\mu}+\left(\mathfrak{A}_{\mu}^{0} e_{0}+\mathfrak{A}_{\mu}^{a} e_{a}\right) & 0 \\
0 & \partial_{\mu}+\left(\mathfrak{B}_{\mu}^{0} e_{0}+\mathfrak{B}_{\mu}^{a} e_{a}\right)
\end{array}\right)
$$

where e and g represent the electric and magnetic charges of dyons. From equation (24) we get,

$$
\left[\mathscr{D}_{\mu}, \mathscr{D}_{\nu}\right]=\left(\begin{array}{cc}
G_{\mu \nu}^{0} e_{0}+G_{\mu \nu}^{a} e_{a} & 0 \\
0 & \mathscr{G}_{\mu \nu}^{0} e_{0}+\mathscr{G}_{\mu \nu}^{a} e_{a}
\end{array}\right)
$$

which is $U(1) \times S U(2)$ octonion gauge field strength for dyons in terms of $2 \times 2$ Zorn matrix vector realization. Equation (25) may be reduced to following compact form as

$$
\left[\mathscr{D}_{\mu}, \mathscr{D}_{\nu}\right]=\mathbb{G}_{\mu \nu}
$$

where

$$
\mathbb{G}_{\mu \nu}=\left(\begin{array}{cc}
G_{\mu}^{\mathrm{e}} & 0 \\
0 & \mathscr{G}_{\mu}^{\mathrm{g}}
\end{array}\right) \simeq\left(\begin{array}{cc}
G_{\mu \nu}^{0} e_{0}+G_{\mu \nu}^{a} e_{a} & 0 \\
0 & \mathscr{G}_{\mu \nu}^{0} e_{0}+\mathscr{G}_{\mu \nu}^{a} e_{a}
\end{array}\right)
$$

with

$$
\left\{\begin{array}{l}
G_{\mu \nu}^{0}=\partial_{\mu} \mathfrak{A}_{\nu}^{0}-\partial_{\nu} \mathfrak{A}_{\mu}^{0}+e_{0}\left[\mathfrak{A}_{\mu}^{0}, \mathfrak{A}_{\nu}^{0}\right] \\
G_{\mu \nu}^{a}=\partial_{\mu} \mathfrak{A}_{\nu}^{a}-\partial_{\nu} \mathfrak{A}_{\mu}^{a}+e_{a}\left[\mathfrak{A}_{\mu}^{a}, \mathfrak{A}_{\nu}^{a}\right]
\end{array}\right.
$$


Here $G_{\mu \nu}^{0}$ and $G_{\mu \nu}^{a}$ respectively describe the abelian and non-Abelian, i.e. $U(1)_{e} \times S U(2)_{e}$ electroweak gauge structures in presence of electric charge, while

$$
\left\{\begin{array}{l}
\mathscr{G}_{\mu \nu}^{0}=\partial_{\mu} \mathfrak{B}_{\nu}^{0}-\partial_{\nu} \mathfrak{B}_{\mu}^{0}+e_{0}\left[\mathfrak{B}_{\mu}^{0}, \mathfrak{B}_{\nu}^{0}\right] \\
\mathscr{G}_{\mu \nu}^{a}=\partial_{\mu} \mathfrak{B}_{\nu}^{a}-\partial_{\nu} \mathfrak{B}_{\mu}^{a}+e_{a}\left[\mathfrak{B}_{\mu}^{a}, \mathfrak{B}_{\nu}^{a}\right]
\end{array}\right.
$$

describes the $U(1)_{m} \times S U(2)_{m}$ electroweak gauge structure due to the presence of magnetic monopole. From equations (28) and (29), we get the following gauge field strength of dyons as

$$
\left(\begin{array}{c}
G_{\mu \nu}^{0} \\
G_{\mu \nu}^{a} \\
\mathscr{G}_{\mu \nu}^{0} \\
\mathscr{G}_{\mu \nu}^{a}
\end{array}\right) \Longrightarrow\left(\begin{array}{c}
\mathcal{E}_{\mu \nu}^{0} \\
\mathcal{E}_{\mu \nu}^{a} \\
\mathcal{H}_{\mu \nu}^{0} \\
\mathcal{H}_{\mu \nu}^{a}
\end{array}\right) .
$$

Here $\mathcal{E}_{\mu \nu}^{0}$ and $\mathcal{H}_{\mu \nu}^{0}$ are defined as the electric and magnetic field tensors of dyons for the case of $U(1)$ while $\mathcal{E}_{\mu}^{a}$ and $\mathcal{H}_{\mu}^{a}$ describe the $S U(2)$ non-Abelian gauge field strength. Operating $\mathscr{D}_{\mu}$ given by the equation (24) to the $U(1) \times S U(2)$ octonion gauge field strength $\mathbb{G}_{\mu \nu}$ (27), we get

$$
\mathscr{D}_{\mu} \mathbb{G}_{\mu \nu}=\left(\begin{array}{cc}
\partial_{\mu} G_{\mu \nu}^{0} e_{0}+\partial_{\mu} G_{\mu \nu}^{a} e_{a} & 0 \\
0 & \partial_{\mu} \mathscr{G}_{\mu \nu}^{0} e_{0}+\partial_{\mu} \mathscr{G}_{\mu \nu}^{a} e_{a}
\end{array}\right),
$$

which may further be reduced in terms of compect notation of split octonion formulation, i.e.

$$
\mathscr{D}_{\mu} \mathbb{G}_{\mu \nu}=\mathbb{J}_{\nu} \Longrightarrow\left(\begin{array}{cc}
\mathbb{J}_{\nu}^{\mathrm{e}} & 0 \\
0 & \mathbb{J}_{\nu}^{\mathrm{g}}
\end{array}\right) \simeq\left(\begin{array}{cc}
\mathrm{j}_{\nu}^{0} e_{0}+\mathrm{j}_{\nu}^{a} e_{a} & 0 \\
0 & \mathrm{k}_{\nu}^{0} e_{0}+\mathrm{k}_{\nu}^{a} e_{a}
\end{array}\right)
$$

Here $\mathbb{J}_{\nu}$ is $U(1) \times S U(2)$ form of octonion gauge current familiar to normal weak current for dyons. From equation (32), we may write the following field equations

$$
\left\{\begin{array}{l}
\mathrm{j}_{\nu}^{0}=\partial_{\mu} G_{\mu \nu}^{0} ; \\
\mathrm{j}_{\nu}^{a}=\partial_{\mu} G_{\mu \nu}^{a} ; \\
\mathrm{k}_{\nu}^{0}=\partial_{\mu} \mathscr{G}_{\mu \nu}^{0} ; \\
\mathrm{k}_{\nu}^{a}=\partial_{\mu} \mathscr{G}_{\mu \nu}^{a} ;
\end{array}\right.
$$

where $\mathrm{j}_{\nu}^{0}$ and $\mathrm{j}_{\nu}^{a}$ are generalized octonion electroweak current for $U(1)_{e} \times S U(2)_{e}$ (electric case) while $\mathrm{k}_{\nu}^{0}$ and $\mathrm{k}_{\nu}^{a}$ for $U(1)_{m} \times S U(2)_{m}$ (magnetic case). Using equation (30), we may obtain the following gauge field equations of dyons for the case of electroweak gauge formulation,

$$
\left\{\begin{array}{l}
\mathrm{j}_{\nu}^{0}=\partial_{\mu} \mathcal{E}_{\mu \nu}^{0} ; \\
\mathrm{j}_{\nu}^{a}=\partial_{\mu} \mathcal{E}_{\mu \nu}^{a} ; \\
\mathrm{k}_{\nu}^{0}=\partial_{\mu} \mathcal{H}_{\mu \nu}^{0} ; \\
\mathrm{k}_{\nu}^{a}=\partial_{\mu} \mathcal{H}_{\mu \nu}^{a} ;
\end{array}\right.
$$


So, the analogous continuity equation then changes to be

$$
\mathscr{D}_{\mu} \mathbb{J}_{\mu}=\left(\begin{array}{cc}
\partial_{\mu} \mathrm{j}_{\mu}^{0} e_{0}+\partial_{\mu} \mathrm{j}_{\mu}^{a} e_{a} & 0 \\
0 & \partial_{\mu} \mathrm{k}_{\nu}^{0} e_{0}+\partial_{\mu} \mathrm{k}_{\nu}^{a} e_{a}
\end{array}\right)=0 .
$$

Hence, the split octonion formulation of electroweak gauge theory is consistent, compect and simpler.

\section{Octonionic formulation of Grand Unified Theory}

Keeping in mind the role of octonion in gauge field theories, let us start with the local $S U(3) \times$ $S U(2) \times U(1)$ gauge symmetry which is an internal symmetry representing the Standard Model (SM). The smallest simple Lie group which contains the standard model, and upon which the first Grand Unified Theory (GUT) was based, is $S U(5) \supset S U(3) \times S U(2) \times U(1)$. Thus we may extend $S U(2) \times U(1)$ electroweak gauge theory to the $S U(3) \times S U(2) \times U(1)$ gauge theory $(\mathrm{GUT})$ in terms of split octonion formulation. We may now write the $S U(3) \times S U(2) \times U(1)$ gauge field for generalized fields of dyons as

$$
\begin{aligned}
\left(\begin{array}{c}
\mathfrak{A}_{\mu} \\
\mathfrak{B}_{\mu}
\end{array}\right) \longmapsto & \left(\begin{array}{c}
\mathfrak{A}_{\mu}^{0} e_{0}+\mathfrak{A}_{\mu}^{a} e_{a}+\mathfrak{A}_{\mu}^{\alpha} e_{\alpha} \\
\mathfrak{B}_{\mu}^{0} e_{0}+\mathfrak{B}_{\mu}^{a} e_{a}+\mathfrak{B}_{\mu}^{\alpha} e_{\alpha}
\end{array}\right), \\
& (\forall \mu=0,1,2,3 ; \quad a=1,2,3 ; \quad \alpha=1,2, \ldots \ldots, 8)
\end{aligned}
$$

where the components of electric $\mathfrak{A}_{\mu}^{0}$ and magnetic $\mathfrak{B}_{\mu}^{0}$ are the four potentials of dyons in case of $U(1)$. Similarly, the $\mathfrak{A}_{\mu}^{a}, \mathfrak{B}_{\mu}^{a}$ and $\mathfrak{A}_{\mu}^{\alpha}, \mathfrak{B}_{\mu}^{\alpha}$ describe the $S U(2)$ and $S U(3)$ gauge field strengths for dyons in terms of split octonions. So, the covariant derivative for $S U(3) \times S U(2) \times U(1)$ octonion gauge field in terms of $2 \times 2$ Zorn's vector matrix realization of split octonions is then described as

$$
\mathscr{D}_{\mu}=\left(\begin{array}{cc}
\partial_{\mu}+\left(\mathfrak{A}_{\mu}^{0} e_{0}+\mathfrak{A}_{\mu}^{a} e_{a}+\mathfrak{A}_{\mu}^{\alpha} e_{\alpha}\right) & 0 \\
0 & \partial_{\mu}+\left(\mathfrak{B}_{\mu}^{0} e_{0}+\mathfrak{B}_{\mu}^{a} e_{a}+\mathfrak{B}_{\mu}^{\alpha} e_{\alpha}\right)
\end{array}\right),
$$

which is further reduced to

$$
\left[\mathscr{D}_{\mu}, \mathscr{D}_{\nu}\right]=\left(\begin{array}{cc}
G_{\mu \nu}^{0} e_{0}+G_{\mu \nu}^{a} e_{a}+G_{\mu \nu}^{\alpha} e_{\alpha} & 0 \\
0 & \mathscr{G}_{\mu \nu}^{0} e_{0}+\mathscr{G}_{\mu \nu}^{a} e_{a}+\mathscr{G}_{\mu \nu}^{\alpha} e_{\alpha}
\end{array}\right)
$$

where $G_{\mu \nu}^{0}, G_{\mu \nu}^{a}, G_{\mu \nu}^{\alpha}$ are defined as the $S U(3) \times S U(2) \times U(1)$ grand-unified gauge field strengths of dyons in presence of electric charge, whereas $\mathscr{G}_{\mu \nu}^{0}, \mathscr{G}_{\mu \nu}^{a}, \mathscr{G}_{\mu \nu}^{\alpha}$ describe the $S U(3) \times S U(2) \times U(1)$ grand-unified gauge field strengths in presence of magnetic monopole. Hence, equation (38) take the following forms

$$
\left[\mathscr{D}_{\mu}, \mathscr{D}_{\nu}\right]=\mathbb{G}_{\mu \nu}^{\alpha}, \quad(\forall \alpha=1,2, \ldots \ldots, 8)
$$


which is $S U(3) \times S U(2) \times U(1)$ octonion gauge field strength for dyons. Thus, from equation (39), we identity the octonion gauge field components of $S U(3) \times S U(2) \times U(1)$ are

$$
\left\{\begin{array}{l}
G_{\mu \nu}^{0}=\partial_{\mu} \mathfrak{A}_{\nu}^{0}-\partial_{\nu} \mathfrak{A}_{\mu}^{0}+e_{0}\left[\mathfrak{A}_{\mu}^{0}, \mathfrak{A}_{\nu}^{0}\right] \\
G_{\mu \nu}^{a}=\partial_{\mu} \mathfrak{A}_{\nu}^{a}-\partial_{\nu} \mathfrak{A}_{\mu}^{a}+e_{a}\left[\mathfrak{A}_{\mu}^{a}, \mathfrak{A}_{\nu}^{a}\right] \\
G_{\mu \nu}^{\alpha}=\partial_{\mu} \mathfrak{A}_{\nu}^{\alpha}-\partial_{\nu} \mathfrak{A}_{\mu}^{\alpha}+e_{\alpha}\left[\mathfrak{A}_{\mu}^{\alpha}, \mathfrak{A}_{\nu}^{\alpha}\right]
\end{array}\right.
$$

for $U(1)_{e} \times S U(2)_{e} \times S U(3)_{e}$ grand unified gauge structures in presence of electric charge, and

$$
\left\{\begin{array}{l}
\mathscr{G}_{\mu \nu}^{0}=\partial_{\mu} \mathfrak{B}_{\nu}^{0}-\partial_{\nu} \mathfrak{B}_{\mu}^{0}+e_{0}\left[\mathfrak{B}_{\mu}^{0}, \mathfrak{B}_{\nu}^{0}\right] \\
\mathscr{G}_{\mu \nu}^{a}=\partial_{\mu} \mathfrak{B}_{\nu}^{a}-\partial_{\nu} \mathfrak{B}_{\mu}^{a}+e_{a}\left[\mathfrak{B}_{\mu}^{a}, \mathfrak{B}_{\nu}^{a}\right] \\
\mathscr{G}_{\mu \nu}^{\alpha}=\partial_{\mu} \mathfrak{B}_{\nu}^{\alpha}-\partial_{\nu} \mathfrak{B}_{\mu}^{\alpha}+e_{\alpha}\left[\mathfrak{B}_{\mu}^{\alpha}, \mathfrak{B}_{\nu}^{\alpha}\right]
\end{array}\right.
$$

are the constituents of $U(1)_{m} \times S U(2)_{m} \times S U(3)_{m}$ gauge structure in presence of magnetic monopole. Thus, octonionic formulation leads the following gauge field strengths of dyons as

$$
\left(\begin{array}{l}
G_{\mu \nu}^{0} \\
G_{\mu \nu}^{a} \\
G_{\mu \nu}^{\alpha}
\end{array}\right) \Rightarrow\left(\begin{array}{c}
\mathcal{E}_{\mu \nu}^{0} \\
\mathcal{E}_{\mu \nu}^{a} \\
\mathcal{E}_{\mu \nu}^{\alpha}
\end{array}\right), \quad \text { and }\left(\begin{array}{c}
\mathscr{G}_{\mu \nu}^{0} \\
\mathscr{G}_{\mu \nu}^{a} \\
\mathscr{G}_{\mu \nu}^{\alpha}
\end{array}\right) \Rightarrow\left(\begin{array}{c}
\mathcal{H}_{\mu \nu}^{0} \\
\mathcal{H}_{\mu \nu}^{a} \\
\mathcal{H}_{\mu \nu}^{\alpha}
\end{array}\right)
$$

where $\mathcal{E}_{\mu \nu}^{0}, \mathcal{E}_{\mu \nu}^{a}, \mathcal{E}_{\mu \nu}^{\alpha}$ are respectively defined the electric gauge structures for the case of $U(1)$, $S U(2), S U(3)$ gauge field theory, while $\mathcal{H}_{\mu \nu}^{0}, \mathcal{H}_{\mu \nu}^{a}, \mathcal{H}_{\mu \nu}^{\alpha}$ are described for magnetic monopole gauge structures of dyons. Now we operate $\mathscr{D}_{\mu}$ given by the equation (37) to the octonion gauge field strength $\mathbb{G}_{\mu \nu}^{\alpha}(39)$, we get

$$
\mathscr{D}_{\mu} \mathbb{G}_{\mu \nu}^{\alpha}=\left(\begin{array}{cc}
\partial_{\mu} G_{\mu \nu}^{0} e_{0}+\partial_{\mu} G_{\mu \nu}^{a} e_{a}+\partial_{\mu} G_{\mu \nu}^{\alpha} e_{\alpha} & 0 \\
0 & \partial_{\mu} \mathscr{G}_{\mu \nu}^{0} e_{0}+\partial_{\mu} \mathscr{G}_{\mu \nu}^{a} e_{a}+\partial_{\mu} \mathscr{G}_{\mu \nu}^{\alpha} e_{\alpha}
\end{array}\right),
$$

which may further be reduced in terms of compect notation of octonion form as

$$
\mathscr{D}_{\mu} \mathbb{G}_{\mu \nu}^{\alpha}=\mathbb{J}_{\nu}^{\alpha}
$$

Here $\mathbb{J}_{\nu}^{\alpha}$ is $U(1) \times S U(2) \times S U(3)$ grand-unified form of octonion gauge current for dyons which may be expressed in terms of $2 \times 2$ Zorn vector matrix realization of split octonions as

$$
\mathbb{J}_{\nu}^{\alpha}=\left(\begin{array}{cc}
\mathrm{j}_{\nu}^{0} e_{0}+\mathrm{j}_{\nu}^{a} e_{a}+\mathrm{j}_{\nu}^{\alpha} e_{\alpha} & 0 \\
0 & \mathrm{k}_{\nu}^{0} e_{0}+\mathrm{k}_{\nu}^{a} e_{a}+\mathrm{k}_{\nu}^{\alpha} e_{\alpha}
\end{array}\right) .
$$


The octonionic formulation (44) thus leads to the following field equations of dyons

$$
\left\{\begin{array}{l}
\mathrm{j}_{\nu}^{0}=\partial_{\mu} \mathcal{E}_{\mu \nu}^{0} ; \quad(\forall \mu, \nu=0,1,2,3) \\
\mathrm{j}_{\nu}^{a}=\partial_{\mu} \mathcal{E}_{\mu \nu}^{a} ; \quad(\forall a=1,2,3) \\
\mathrm{j}_{\nu}^{\alpha}=\partial_{\mu} \mathcal{E}_{\mu \nu}^{\alpha} ; \quad(\forall \alpha=1,2,3, \ldots, 8) \\
\mathrm{k}_{\nu}^{0}=\partial_{\mu} \mathcal{H}_{\mu \nu}^{0} ; \quad(\forall \mu, \nu=0,1,2,3) \\
\mathrm{k}_{\nu}^{a}=\partial_{\mu} \mathcal{H}_{\mu \nu}^{a} ; \quad(\forall a=1,2,3) \\
\mathrm{k}_{\nu}^{\alpha}=\partial_{\mu} \mathcal{H}_{\mu \nu}^{\alpha} ; \quad(\forall \alpha=1,2,3, \ldots, 8)
\end{array}\right.
$$

were $\mathrm{j}_{\nu}^{0}$ is the $U(1)$ current for electric charge, $\mathrm{j}_{\nu}^{a}$ is the $S U(2)$ week current associated with electric charge and $\mathrm{j}_{\nu}^{\alpha}$ is the current associated with $S U(3)_{c}$ used for chromo electric charge (i.e. normal color). On the other hand, $\mathrm{k}_{\nu}^{0}$ is $U(1)$ the counterpart of the four current, $\mathrm{k}_{\nu}^{a}$ is the $S U(2)$ weak current while the $\mathrm{k}_{\nu}^{\alpha}$ is $S U(3)_{c}$ gluonic current due to the presence of magnetic monopole (chromo-magnetic charges or magnetic color). As such, the octonionic formulation, regardless a generalization of GUTs for the mixing of gauge currents used for $U(1), S U(2)$ and $S U(3)_{c}$ sectors associated respectively with the electromagnetic, weak and strong interactions in presence of dyons, also shows the duality invariance as well. Consequently, the continuity equation is generalized as

$$
\mathscr{D}_{\mu} \mathbb{J}_{\mu}^{\alpha}=\left(\begin{array}{cc}
\partial_{\mu} \mathrm{j}_{\mu}^{0} e_{0}+\partial_{\mu} \mathrm{j}_{\mu}^{a} e_{a}+\partial_{\mu} \mathrm{j}_{\mu}^{\alpha} e_{\alpha} & 0 \\
0 & \partial_{\mu} \mathrm{k}_{\nu}^{0} e_{0}+\partial_{\mu} \mathrm{k}_{\nu}^{a} e_{a}+\partial_{\mu} \mathrm{k}_{\nu}^{\alpha} e_{\alpha}
\end{array}\right)=0 .
$$

which leading to the conservation of Noetherian current.

\section{Octonion formulation with linear gravity}

Here we assume the octonion eight dimensional space, combination of two quaternionic spaces respectively associated with the electromagnetic interaction (EM-space) and linear gravitational interaction (G-space) [35, 36, 37]. Thus, we may write the split octonionic unified representation of gravitational-electromagnetic (G-EM) space in terms of following $2 \times 2$ Zorn's vector matrix realization as

$$
\begin{aligned}
\mathcal{O} & =\left(\mathcal{O}_{\text {em-space }}, \mathcal{O}_{\text {g-space }}\right) \\
& =\mathcal{O}_{e m}^{0} u_{0}^{*}+\mathcal{O}_{g}^{0} u_{0}+\mathcal{O}_{e m}^{j} u_{j}^{*}+\mathcal{O}_{g}^{j} u_{j} \simeq\left(\begin{array}{cc}
\mathcal{O}_{e m}^{0} e_{0} & -\mathcal{O}_{e m}^{j} e_{j} \\
\mathcal{O}_{g}^{j} e_{j} & \mathcal{O}_{g}^{0} e_{0}
\end{array}\right) \cdot(\forall j=1,2,3)
\end{aligned}
$$

As such, any physical quantity $\mathcal{X} \in \mathcal{O}$ may be written as

$$
\mathcal{X}=\left(\mathcal{X}_{e m}, \mathcal{X}_{g}\right)=\mathcal{X}_{e m}^{0} u_{0}^{*}+\mathcal{X}_{g}^{0} u_{0}+\mathcal{X}_{e m}^{j} u_{j}^{*}+\mathcal{X}_{g}^{j} u_{j} \simeq\left(\begin{array}{cc}
\mathcal{X}_{e m}^{0} e_{0} & -\mathcal{X}_{e m}^{j} e_{j} \\
\mathcal{X}_{g}^{j} e_{j} & \mathcal{X}_{g}^{0} e_{0}
\end{array}\right)
$$


and accordingly the split octonion differential operator $\mathfrak{D}$ may also be written for unified picture of the EM-G space [30] in terms of $2 \times 2$ Zorn's matrix realization [23, 25], i.e.

$$
\mathfrak{D}=\left(\mathfrak{D}_{e m}, \mathfrak{D}_{g}\right) \simeq\left(\begin{array}{cc}
\partial_{e m}^{0} e_{0} & -\partial_{e m}^{j} e_{j} \\
\partial_{g}^{j} e_{j} & -\partial_{g}^{0} e_{0}
\end{array}\right)
$$

while the octonion conjugate of equation (50) is described as

$$
\overline{\mathfrak{D}}=\left(\overline{\mathfrak{D}}_{e m}, \overline{\mathfrak{D}}_{g}\right) \simeq\left(\begin{array}{cc}
-\partial_{e m}^{0} e_{0} & \partial_{e m}^{j} e_{j} \\
-\partial_{g}^{j} e_{j} & \partial_{g}^{0} e_{0}
\end{array}\right) .
$$

Like wise, the split octonion valued potential $\mathscr{V}$ for the unified EM-G space is defined as

$$
\begin{aligned}
\mathscr{V}=\left(\mathscr{V}_{-}, \mathscr{V}_{+}\right) & =\mathscr{V}_{-}^{0} u_{0}^{*}+\mathscr{V}_{+}^{0} u_{0}+\mathscr{V}_{-}^{j} u_{j}^{*}+\mathscr{V}_{+}^{j} u_{j} \\
& =\left(\begin{array}{cc}
\mathscr{V}_{-}^{0} e_{0} & -\mathscr{V}_{+}^{j} e_{j} \\
\mathscr{V}_{-}^{j} e_{j} & \mathscr{V}_{+}^{0} e_{0}
\end{array}\right),
\end{aligned}
$$

where we have expressed the split octonion valued potential in terms two four potential form as the combination of linear gravitational potential $\left(\mathscr{V}_{g}^{\mu}\right)$ and electromagnetic potential $\left(\mathscr{V}_{e m}^{\mu}\right)$ for unified EM-G space, i.e.

$$
\mathscr{V}=\left(\mathscr{V}_{g}, \mathscr{V}_{e m}\right) \simeq\left(\begin{array}{cc}
\left(\mathscr{V}_{g}^{0}-\mathscr{V}_{\text {em }}^{0}\right) e_{0} & -\left(\mathscr{V}_{e m}^{j}+\mathscr{V}_{g}^{j}\right) e_{j} \\
\left(\mathscr{V}_{e m}^{j}-\mathscr{V}_{g}^{j}\right) e_{j} & \left(\mathscr{V}_{g}^{0}+\mathscr{V}_{e m}^{0}\right) e_{0}
\end{array}\right)
$$

where $\mathscr{V}_{g}^{\mu}=\left(\mathscr{V}_{g}^{0}, \mathscr{V}_{g}^{j}\right)$ and $\mathscr{V}_{e m}^{\mu}=\left(\mathscr{V}_{e m}^{0}, \mathscr{V}_{e m}^{j}\right)(\mu=0,1,2,3)$ are respectively denoted as linear gravitational (G-space) and electromagnetic (EM-space) four potentials. Thus, we may write the split octonion potential wave equation for unified EM-G space by operating $\overline{\mathfrak{D}}$ given by equation (51) to octonion potential $\mathscr{V}$ (53) in the following manner,

$$
\overline{\mathfrak{D}} \mathscr{V}=\left(\begin{array}{cc}
{\left[-\left(\partial_{e m}^{0} \mathscr{V}_{g}^{0}+\partial_{e m}^{j} \mathscr{V}_{g}^{j}\right)+\right.} & {\left[\left(\partial_{e m}^{k} \mathscr{V}_{g}^{0}+\partial_{e m}^{0} \mathscr{V}_{g}^{k}+\epsilon_{i j k} \partial_{g}^{i} \mathscr{V}_{e m}^{j}\right)+\right.} \\
\left.\left(\partial_{e m}^{0} \mathscr{V}_{e m}^{0}+\partial_{e m}^{j} \mathscr{V}_{e m}^{j}\right)\right] e_{0} & \left.\left(\partial_{e m}^{k} \mathscr{V}_{e m}^{0}+\partial_{e m}^{0} \mathscr{V}_{e m}^{k}-\epsilon_{i j k} \partial_{g}^{i} \mathscr{V}_{g}^{j}\right)\right] e_{k} \\
{\left[\left(-\partial_{g}^{k} \mathscr{V}_{g}^{0}-\partial_{g}^{0} \mathscr{V}_{g}^{k}+\epsilon_{i j k} \partial_{e m}^{i} \mathscr{V}_{e m}^{j}\right)+\right.} & {\left[\left(\partial_{g}^{0} \mathscr{V}_{g}^{0}+\partial_{g}^{j} \mathscr{V}_{g}^{j}\right)+\right.} \\
\left.\left(\partial_{g}^{k} \mathscr{V}_{e m}^{0}+\partial_{g}^{0} \mathscr{V}_{e m}^{k}+\epsilon_{i j k} \partial_{e m}^{i} \mathscr{V}_{g}^{j}\right)\right] e_{k} & \left.\left(\partial_{g}^{0} \mathscr{V}_{e m}^{0}+\partial_{g}^{j} \mathscr{V}_{e m}^{j}\right)\right] e_{0}
\end{array}\right)
$$

which can further be reduced to

$$
\begin{aligned}
\overline{\mathfrak{D}} \mathscr{V} & =\mathscr{F} \simeq \mathscr{F}_{-}^{0} u_{0}^{*}+\mathscr{F}_{+}^{0} u_{0}+\mathscr{F}_{-}^{j} u_{j}^{*}+\mathscr{F}_{+}^{j} u_{j} \\
& =\left(\begin{array}{cc}
\mathscr{F}_{-}^{0} e_{0} & -\mathscr{F}_{+}^{j} e_{j} \\
\mathscr{F}_{-}^{j} e_{j} & \mathscr{F}_{+}^{0} e_{0}
\end{array}\right) \simeq\left(\begin{array}{cc}
\left(\mathscr{F}_{g}^{0}-\mathscr{F}_{e m}^{0}\right) e_{0} & -\left(\mathscr{F}_{e m}^{j}+\mathscr{F}_{g}^{j}\right) e_{j} \\
\left(\mathscr{F}_{e m}^{j}-\mathscr{F}_{g}^{j}\right) e_{j} & \left(\mathscr{F}_{g}^{0}+\mathscr{F}_{e m}^{0}\right) e_{0}
\end{array}\right),
\end{aligned}
$$


where $\mathscr{F}$ is an split octonion which reproduces the field strength of generalized gravitationalelectromagnetic interactions of dyons. Thus the unified components of $\mathscr{F}$ are expressed as

$$
\begin{aligned}
\left(\partial_{e m}^{0} \mathscr{V}_{g}^{0}+\partial_{e m}^{j} \mathscr{V}_{g}^{j}\right) e_{0} & =\mathscr{F}_{(e m-g)}^{j} ; \\
\left(\partial_{e m}^{0} \mathscr{V}_{e m}^{0}+\partial_{e m}^{j} \mathscr{V}_{e m}^{j}\right) e_{0} & =\mathscr{F}_{(e m-e m)}^{j} ; \\
\left(\partial_{e m}^{k} \mathscr{V}_{g}^{0}+\partial_{e m}^{0} \mathscr{V}_{g}^{k}+\epsilon_{i j k} \partial_{g}^{i} \mathscr{V}_{e m}^{j}\right) e_{k} & =\mathscr{F}_{(e m-g) /(g-e m)}^{k} ; \\
\left(\partial_{e m}^{k} \mathscr{V}_{e m}^{0}+\partial_{e m}^{0} \mathscr{V}_{e m}^{k}-\epsilon_{i j k} \partial_{g}^{i} \mathscr{V}_{g}^{j}\right) e_{k} & =\mathscr{F}_{(e m-e m) /(g-g)}^{k} ; \\
\left(-\partial_{g}^{k} \mathscr{V}_{g}^{0}-\partial_{g}^{0} \mathscr{V}_{g}^{k}+\epsilon_{i j k} \partial_{e m}^{i} \mathscr{V}_{e m}^{j}\right) e_{k} & =\mathscr{F}_{(g-g) /(e m-e m)}^{k} ; \\
\left(\partial_{g}^{k} \mathscr{V}_{e m}^{0}+\partial_{g}^{0} \mathscr{V}_{e m}^{k}+\epsilon_{i j k} \partial_{e m}^{i} \mathscr{V}_{g}^{j}\right) e_{k} & =\mathscr{F}_{(g-e m) /(e m-g)}^{k} ; \\
\left(\partial_{g}^{0} \mathscr{V}_{g}^{0}+\partial_{g}^{j} \mathscr{V}_{g}^{j}\right) e_{0} & =\mathscr{F}_{(g-g)}^{j} ; \\
\left(\partial_{g}^{0} \mathscr{V}_{e m}^{0}+\partial_{g}^{j} \mathscr{V}_{e m}^{j}\right) e_{0} & =\mathscr{F}_{(g-e m)}^{j} .
\end{aligned}
$$

Here the terms $\left(\mathscr{F}_{e m-e m}^{j}, \mathscr{F}_{g-g}^{j}\right)$ are defined the interactions of electromagnetic- electromagnetic (EM-EM), gravitational- gravitational (G-G) field strength, and $\mathscr{F}_{(e m-g)}^{j}, \mathscr{F}_{g-e m}^{j}$ are described as the combinations of electromagnetic- gravitational (EM-G), gravitational- electromagnetic (G-EM) field strength while the other components $\mathscr{F}_{(e m-g) /(g-e m)}^{k}, \mathscr{F}_{(e m-e m) /(g-g)}^{k}$, $\mathscr{F}_{(g-g) /(e m-e m)}^{k}, \mathscr{F}_{(g-e m) /(e m-g)}^{k}$ are defined the combined interactions of electromagnetic and gravitational for octonion space. Applied the following Lorentz Gauge conditions

$$
\begin{aligned}
\left(\partial_{e m}^{0} \mathscr{V}_{g}^{0}+\partial_{e m}^{j} \mathscr{V}_{g}^{j}\right) & =0 ; \\
\left(\partial_{e m}^{0} \mathscr{V}_{e m}^{0}+\partial_{e m}^{j} \mathscr{V}_{e m}^{j}\right) & =0 ; \\
\left(\partial_{g}^{0} \mathscr{V}_{g}^{0}+\partial_{g}^{j} \mathscr{V}_{g}^{j}\right) & =0 ; \\
\left(\partial_{g}^{0} \mathscr{V}_{e m}^{0}+\partial_{g}^{j} \mathscr{V}_{e m}^{j}\right) & =0 ;
\end{aligned}
$$

we get the following split octonion form of the field tensor associated with the unified gravitationalelectromagnetic (G-EM) interactions as

$$
\mathscr{F}=\left(\mathscr{F}_{e m}^{j}-\mathscr{F}_{g}^{j}\right) u_{j}^{*}+\left(\mathscr{F}_{e m}^{j}+\mathscr{F}_{g}^{j}\right) u_{j}=\left(\begin{array}{cc}
0 & -\left(\mathscr{F}_{e m}^{j}+\mathscr{F}_{g}^{j}\right) e_{j} \\
\left(\mathscr{F}_{e m}^{j}-\mathscr{F}_{g}^{j}\right) e_{j} & 0
\end{array}\right) .
$$

which can further be reduced to

$$
\mathscr{F}=\left(\begin{array}{cc}
0 & -\Psi_{(e m-g)_{+}}^{j} e_{j} \\
\Psi_{(e m-g)_{-}}^{j} e_{j} & 0
\end{array}\right)
$$

where $\Psi_{(e m-g)_{+}}^{j} \rightarrow\left(\mathscr{F}_{e m}^{j}+\mathscr{F}_{g}^{j}\right)$ and $\Psi_{(e m-g)_{-}}^{j} \rightarrow\left(\mathscr{F}_{e m}^{j}-\mathscr{F}_{g}^{j}\right)$ are generalized EM-G fields in octonionic space. Hence, we get the split octonionic field equation in unified gravitationalelectromagnetic space on applying the differential operator (50) to equation (58) as

$$
\mathfrak{D} \mathscr{F}=\mathcal{J},
$$


where $\mathcal{J}$ is the octonionic form of unified gravitational-electromagnetic field current defined as

$$
\mathcal{J}=\left(\begin{array}{cc}
\left(\mathfrak{J}_{(e m-g)}-\mathfrak{J}_{(e m-e m)}\right) e_{0} & -\left(\mathfrak{J}_{(e m-e m) /(g-g)}-\mathfrak{J}_{(e m-g) /(g-e m)}\right) e_{j} \\
\left(\mathfrak{J}_{(g-g) /(e m-e m)}+\mathfrak{J}_{(g-e m) /(e m-g)}\right) e_{j} & \left(\mathfrak{J}_{(g-e m)}-\mathfrak{J}_{(g-g)}\right) e_{0}
\end{array}\right)
$$

Here $\left(\mathfrak{J}_{(e m-g)}, \mathfrak{J}_{(e m-e m)}\right)$, and $\left(\mathfrak{J}_{(g-e m)}, \mathfrak{J}_{(g-g)}\right)$ are described as the octonionic current source density respectively for (EM-G, EM-EM) and (G-EM, G-G) spaces, while other components $\left(\mathfrak{J}_{(e m-e m) /(g-g)}, \mathfrak{J}_{(e m-g) /(g-e m)}\right)$ and $\left(\mathfrak{J}_{(g-g) /(e m-e m)}, \mathfrak{J}_{(g-e m) /(e m-g)}\right)$ are the mixed current source densities of (EM-EM/G-G, EM-G/ G-EM) and (G-G/EM-EM, G-EM/EM-G) octonionic spaces [30]. Thus from equation (60), we get

$$
\mathfrak{D} \mathscr{F}=\left(\begin{array}{cc}
\left(\partial_{e m}^{j} \mathscr{F}_{g}^{j}-\partial_{e m}^{j} \mathscr{F}_{e m}^{j}\right) e_{0} & -\left(\partial_{e m}^{0} \mathscr{F}_{e m}^{k}-\epsilon_{i j k} \partial_{g}^{i} \mathscr{F}_{g}^{j}-\right. \\
\left(-\partial_{g}^{0} \mathscr{F}_{e m}^{k}+\epsilon_{i j k} \partial_{e m}^{i} \mathscr{F}_{g}^{j}+\right. & \left.\partial_{e m}^{0} \mathscr{F}_{g}^{k}+\epsilon_{i j k} \partial_{g}^{i} \mathscr{F}_{e m}^{j}\right) e_{k} \\
\left.\partial_{g}^{0} \mathscr{F}_{g}^{k}+\epsilon_{i j k} \partial_{e m}^{i} \mathscr{F}_{e m}^{j}\right) e_{k} & \left(\partial_{g}^{j} \mathscr{F}_{e m}^{j}-\partial_{g}^{j} \mathscr{F}_{g}^{j}\right) e_{0}
\end{array}\right)
$$

where the components of split octonionic current source density $\mathcal{J}$ are described as

$$
\begin{aligned}
\partial_{e m}^{j} \mathscr{F}_{g}^{j} e_{0} & =\mathfrak{J}_{(e m-g)}^{j} ; \\
\partial_{e m}^{j} \mathscr{F}_{e m}^{j} e_{0} & =\mathfrak{J}_{(e m-e m)}^{j} ; \\
\left(\partial_{e m}^{0} \mathscr{F}_{e m}^{k}-\epsilon_{i j k} \partial_{g}^{i} \mathscr{F}_{g}^{j}\right) e_{k} & =\mathfrak{J}_{(e m-e m) /(g-g)}^{k} ; \\
\left(\partial_{e m}^{0} \mathscr{F}_{g}^{k}+\epsilon_{i j k} \partial_{g}^{i} \mathscr{F}_{e m}^{j}\right) e_{k} & =\mathfrak{J}_{(e m-g) /(g-e m)}^{k} ; \\
\left(-\partial_{g}^{0} \mathscr{F}_{e m}^{k}+\epsilon_{i j k} \partial_{e m}^{i} \mathscr{F}_{g}^{j}\right) e_{k} & =\mathfrak{J}_{(g-e m) /(e m-g)}^{k} ; \\
\left(\partial_{g}^{0} \mathscr{F}_{g}^{k}+\epsilon_{i j k} \partial_{e m}^{i} \mathscr{F}_{e m}^{j}\right) e_{k} & =\mathfrak{J}_{(g-g) /(e m-e m)}^{k} ; \\
\partial_{g}^{j} \mathscr{F}_{e m}^{j} e_{0} & =\mathfrak{J}_{(g-e m)}^{j} ; \\
\partial_{g}^{j} \mathscr{F}_{g}^{j} e_{0} & =\mathfrak{J}_{(g-g)}^{j} ;
\end{aligned}
$$

which are the various kinds of fields equations in presence of gravitational-gravitational (G-G), electromagnetic-electromagnetic (EM-EM), electromagnetic-gravitational (EM-G), gravitationalelectromagnetic (G-EM) and their combined interactions.

Moreover, we write the split octonionic radius vector $\mathcal{R}$ as

$$
\mathcal{R}=\mathcal{R}_{e m}^{0} u_{0}^{*}+\mathcal{R}_{g}^{0} u_{0}+\mathcal{R}_{e m}^{j} u_{j}^{*}+\mathcal{R}_{g}^{j} u_{j} \simeq\left(\begin{array}{cc}
\mathcal{R}_{e m}^{0} e_{0} & -\mathcal{R}_{e m}^{j} e_{j} \\
\mathcal{R}_{g}^{j} e_{j} & \mathcal{R}_{g}^{0} e_{0}
\end{array}\right)
$$

which directly reproduces the velocity $(\mathfrak{V})$ of the particle in unified gravitational-electromagnetic space. Furthermore, $\mathfrak{V}$ can be written as the following $2 \times 2$ Zorn's matrix realization of split 
octonion $\left(u_{0}, u_{j}, u_{0}^{*}, u_{j}^{*}\right)$ as

$$
\begin{aligned}
\mathfrak{V} & =\frac{\partial \mathcal{R}}{\partial t}=\frac{\partial}{\partial t}\left\{\mathcal{R}_{e m}^{0} u_{0}^{*}+\mathcal{R}_{g}^{0} u_{0}+\mathcal{R}_{e m}^{j} u_{j}^{*}+\mathcal{R}_{g}^{j} u_{j}\right\} \\
& =\mathfrak{V}_{e m}^{0} u_{0}^{*}+\mathfrak{V}_{g}^{0} u_{0}+\mathfrak{V}_{e m}^{j} u_{j}^{*}+\mathfrak{V}_{g}^{j} u_{j}, \\
& =\left(\begin{array}{cc}
\mathfrak{V}_{e m}^{0} e_{0} & -\mathfrak{V}_{e m}^{j} e_{j} \\
\mathfrak{V}_{g}^{j} e_{j} & \mathfrak{V}_{g}^{0} e_{0}
\end{array}\right) .
\end{aligned}
$$

Hence, the momentum of massive particle also be expressed in the following octonionic form as

$$
\mathscr{P}=\mathscr{P}_{e m}^{0} u_{0}^{*}+\mathscr{P}_{g}^{0} u_{0}+\mathscr{P}_{e m}^{j} u_{j}^{*}+\mathscr{P}_{g}^{j} u_{j}=\left(\begin{array}{cc}
\mathscr{P}_{e m}^{0} e_{0} & -\mathscr{P}_{e m}^{j} e_{j} \\
\mathscr{P}_{g}^{j} e_{j} & \mathscr{P}_{g}^{0} e_{0}
\end{array}\right),
$$

whereas the charge and mass [30 of the particle in octonion formulation may also be analysed as,

$$
\begin{gathered}
\mathfrak{J}_{(e m-e m) /(g-g)}=Q_{(e m-e m) /(g-g)} \mathfrak{V}_{(e m-e m) /(g-g)}, \\
\mathfrak{J}_{(e m-g) /(g-e m)}=Q_{(e m-g) /(g-e m)} \mathfrak{V}_{(e m-g) /(g-e m)}, \\
\mathfrak{J}_{g-g}=Q_{(g-g)} \mathfrak{V}_{(g-g)}, \mathfrak{J}_{(e m-e m)}=Q_{(e m-e m)} \mathfrak{V}_{(e m-e m)},
\end{gathered}
$$

where $Q_{(g-g)}, Q_{(e m-g)}, Q_{(g-e m)}$ and $Q_{(e m-g) /(g-e m)}, Q_{e m-e m) /(g-g)}$ respectively denote the mass of the gravitational- gravitational (G-G), electromagnetic- gravitational (EM-G), gravitationalelectromagnetic (G-EM) interactions etc., while $Q_{(e m-e m)}$ represent the charge of the electromagneticelectromagnetic (EM-EM) interaction. Thus $Q_{(g-g)}, Q_{(e m-g)}, Q_{(g-e m)}$, their other combinations and $Q_{(e m-e m)}$ respectively describe the generalized mass and generalized charge [30, 35, 36, 37] of dyons in the consistent unified picture for split octonions.

\section{Octonion Dark Matter (ODM)}

Dark matter [38, 39] is a type of matter hypothesized to account for a large part of the total mass in the universe. Only about $4.6 \%$ of the mass-energy of the universe is ordinary matter, about $23 \%$ is thought to be composed of dark matter. The remaining $72 \%$ is thought to consist of dark energy, an even stranger component, distributed almost uniformly in space and with energy density non-evolving or slowly evolving with time. On the other hand, the nonbaryonic form of octonionic dark matter [38, 39] is evident through its gravitational effect only.

However, form experiment point of views the summary of the current measurements of the matter density $\Omega_{\text {matter }}$, the dark matter density $\Omega_{D M}$ and the energy density $\Omega_{\text {energy }}$ is already given by Hagiwara [41]. Each are in units of the critical density $\rho_{\text {critical }} \simeq 3 h^{2} /(8 \pi \mathbb{G})$, where $\mathbb{G}$ is the Newton's gravitational constant, and $h$ is the present value of the Hubble constant. The experimental results [42, 43, require the best current values of the matter and energy densities respectively given as $\Omega_{\text {matter }} \simeq 0.27$ and $\Omega_{\text {energy }} \simeq 0.73$. The measurements describe the baryon density $\Omega_{\text {baryon }}$ to a value less than $\sim 0.03$. The difference $\Omega_{\text {matter }}-\Omega_{\text {baryon }} \simeq 0.24$ must be in the form of non-baryonic dark matter. Some experimental results for energy, matter and dark matter densities [44, 45] are shown in Table-2. 


\begin{tabular}{|c|c|}
\hline $0.49 \leq \Omega_{\text {energy }} \leq 0.74$ & $0.49 \leq \Omega_{\text {energy }} \leq 0.76$ \\
\hline $0.20 \leq \Omega_{\text {matter }} \leq 0.50$ & \\
\hline $0.11 \leq h^{2} \Omega_{D M} \leq 0.17$ & $0.09 \leq h^{2} \Omega_{D M} \leq 0.17$ \\
\hline $0.00 \leq h^{2} \Omega_{\text {Hot-dark-matter }} \leq 0.12$ & \\
\hline $0.10 \leq h^{2} \Omega_{\text {Cold-dark-matter }} \leq 0.32$ & \\
\hline $0.02 \leq h^{2} \Omega_{\text {baryon }} \leq 0.03$ & $0.01 \leq h^{2} \Omega_{\text {baryon }} \leq 0.03$ \\
\hline
\end{tabular}

Table 2: The experimental results for Matter and Dark Matter densities with the compression of first release data and newest data.

The ranges of density parameters within the standard cosmological model derived from the first release data with corresponding values newest data and $h \simeq 0.74 \pm 0.08$ is the Hubble parameter [44, 45]. Thus, the matter density:

$$
\begin{aligned}
\Omega_{\text {matter }} & \simeq \Omega_{\text {baryon }}+\Omega_{\text {Hot-dark-matter }}+\Omega_{\text {Cold-dark-matter }} \\
& \simeq \Omega_{\text {baryon }}+\Omega_{D M}
\end{aligned}
$$

includes both baryonic matter and dark matter. Moreover the dark matter can be classed either as hot or cold depending on whether it was relativistic or not in the early universe. So, we can analyse the hot and cold dark matter in terms of split octonions. We assume that the total octonionic dark matter density (ODM) is described as

$$
\Omega_{\mathcal{O}-D M} \simeq \Omega_{\mathcal{O}-H D M}+\Omega_{\mathcal{O}-C D M},
$$

where $\Omega_{\mathcal{O}-H D M}$ describes the octonionic hot dark matter density (OHDM) while $\Omega_{\mathcal{O}-C D M}$ describes the octonionic cold dark matter density (OCDM).

\section{Octonion Hot and Cold Dark matter}

Let us assumed that the octonionic hot dark matter (OHDM) describes the particles that have zero or near-zero masses. Thus we assume that the octonionic hot dark matter (OHDM) is associated with the gravitational-gravitational (G-G), electromagnetic-electromagnetic (EM-EM) and the combination of (G-G) and (EM-EM) fields [30, 35, 36, 37] interactions. So, the hot dark matter may then be described in terms of following $2 \times 2$ Zorn vector matrix realization of split octonions, i.e.

$$
\mathscr{F}_{\mathcal{O}-H D M}=\left(\begin{array}{cc}
0 & \left(\partial_{e m}^{k} \mathscr{V}_{e m}^{0}+\partial_{e m}^{0} \mathscr{V}_{e m}^{k}-\epsilon_{i j k} \partial_{g}^{i} \mathscr{V}_{g}^{j}\right) e_{k} \\
\left(-\partial_{g}^{k} \mathscr{V}_{g}^{0}-\partial_{g}^{0} \mathscr{V}_{g}^{k}+\epsilon_{i j k} \partial_{e m}^{i} \mathscr{V}_{e m}^{j}\right) e_{k} & 0
\end{array}\right)
$$


where $\mathscr{F}_{\mathcal{O}-H D M}$ is an octonionic field strength represents the hot dark matter. So, the components of equation (70) are given as

$$
\left\{\begin{array}{l}
\mathscr{F}_{(e m-e m) /(g-g)}^{k}=\left(\partial_{e m}^{k} \mathscr{V}_{e m}^{0}+\partial_{e m}^{0} \mathscr{V}_{e m}^{k}-\epsilon_{i j k} \partial_{g}^{i} \mathscr{V}_{g}^{j}\right) e_{k} \\
\mathscr{F}_{(g-g) /(e m-e m)}^{k}=\left(-\partial_{g}^{k} \mathscr{V}_{g}^{0}-\partial_{g}^{0} \mathscr{V}_{g}^{k}+\epsilon_{i j k} \partial_{e m}^{i} \mathscr{V}_{e m}^{j}\right) e_{k}
\end{array}\right.
$$

Here the octonion hot dark matter (OHDM) represents the quantas of EM-EM interactions (namely photons) and G-G interactions (i.e. gravitons). Similarly, the required current source density for octonionic hot dark matter (OHDM) is expressed by the following split octonion form as,

$$
\mathcal{J}_{\mathcal{O}-H D M}=\left(\begin{array}{cc}
0 & -\left(\partial_{e m}^{0} \mathscr{F}_{e m}^{k}-\epsilon_{i j k} \partial_{g}^{i} \mathscr{F}_{g}^{j}\right) e_{k} \\
\left(\partial_{g}^{0} \mathscr{F}_{g}^{k}+\epsilon_{i j k} \partial_{e m}^{i} \mathscr{F}_{e m}^{j}\right) e_{k} & 0
\end{array}\right)
$$

which represent the non-baryonic octonion hot dark matter current source density $\mathcal{J}_{\mathcal{O}-H D M}$ associated with massless photons, gravitons, etc. Thus the current source equations for octonionic hot dark matter (OHDM) are given below:

$$
\left\{\begin{array}{l}
\mathfrak{J}_{(e m-e m) /(g-g)}^{k}=\left(\partial_{e m}^{0} \mathscr{F}_{e m}^{k}-\epsilon_{i j k} \partial_{g}^{i} \mathscr{F}_{g}^{j}\right) e_{k} \\
\mathfrak{J}_{(g-g) /(e m-e m)}^{k}=\left(\partial_{g}^{0} \mathscr{F}_{g}^{k}+\epsilon_{i j k} \partial_{e m}^{i} \mathscr{F}_{e m}^{j}\right) e_{k}
\end{array}\right.
$$

Rather, the octonion cold dark matter (OCDM) can be described as the composition of the massive objects moving at sub-relativistic velocities. So, the difference between the octonion cold dark matter (OCDM) and the octonions hot dark matter (OHDM) is significant in the formulation of structure, because the velocities of octonions hot dark matter cause it to wipe out structure on small scales. Thus, the octonions cold dark matter is associated with the electromagnetic-gravitational (EM-G), gravitational-electromagnetic (G-EM) and their mixed interactions. Thus, we analyse the octonion cold dark matter for massive particles, responsible for EM-G and G-EM interactions, i.e.

$$
\mathscr{F}_{\mathcal{O}-C D M}=\left(\begin{array}{cc}
0 & \left(\partial_{e m}^{k} \mathscr{V}_{g}^{0}+\partial_{e m}^{0} \mathscr{V}_{g}^{k}+\epsilon_{i j k} \partial_{g}^{i} \mathscr{V}_{e m}^{j}\right) e_{k} \\
\left(\partial_{g}^{k} \mathscr{V}_{e m}^{0}+\partial_{g}^{0} \mathscr{V}_{e m}^{k}+\epsilon_{i j k} \partial_{e m}^{i} \mathscr{V}_{g}^{j}\right) e_{k} & 0
\end{array}\right)
$$

where $\mathscr{F}_{\mathcal{O}_{-} C D M}$ represents the octonionic field tensor associated with the cold dark matter. Equation (74) reduced to following components

$$
\left\{\begin{array}{l}
\mathscr{F}_{(e m-g) /(g-e m)}^{k}=\left(\partial_{e m}^{k} \mathscr{V}_{g}^{0}+\partial_{e m}^{0} \mathscr{V}_{g}^{k}+\epsilon_{i j k} \partial_{g}^{i} \mathscr{V}_{e m}^{j}\right) e_{k} \\
\mathscr{F}_{(g-e m) /(e m-g)}^{k}=\left(\partial_{g}^{k} \mathscr{V}_{e m}^{0}+\partial_{g}^{0} \mathscr{V}_{e m}^{k}+\epsilon_{i j k} \partial_{e m}^{i} \mathscr{V}_{g}^{j}\right) e_{k}
\end{array}\right.
$$

So, the mediators for octonionic cold dark matter (OCDM), which are non-baryonic particles like $W^{ \pm}, Z^{0}$, etc. Therefore, the current source density for octonionic cold dark matter (OCDM) 
can expressed as

$$
\mathcal{J}_{\mathcal{O}-C D M}=\left(\begin{array}{cc}
\left(\partial_{e m}^{j} \mathscr{F}_{g}^{j}-\partial_{e m}^{j} \mathscr{F}_{e m}^{j}\right) e_{0} & \left.-\partial_{e m}^{0} \mathscr{F}_{g}^{k}+\epsilon_{i j k} \partial_{g}^{i} \mathscr{F}_{e m}^{j}\right) e_{k} \\
\left(-\partial_{g}^{0} \mathscr{F}_{e m}^{k}+\epsilon_{i j k} \partial_{e m}^{i} \mathscr{F}_{g}^{j}\right) e_{k} & \left(\partial_{g}^{j} \mathscr{F}_{e m}^{j}-\partial_{g}^{j} \mathscr{F}_{g}^{j}\right) e_{0}
\end{array}\right),
$$

where $\mathcal{J}_{\mathcal{O}-C D M}$ defines the octonionic cold dark matter current source density, which associated EM-G and EM-G fields spaces. Thus the current source equations for octonionic cold dark matter are:

$$
\begin{cases}\mathfrak{J}_{(e m-g) /(e m-e m)}^{j} & =\left(\partial_{e m}^{j} \mathscr{F}_{g}^{j}-\partial_{e m}^{j} \mathscr{F}_{e m}^{j}\right) ; \\ \mathfrak{J}_{(g-e m) /(g-g)}^{j} & =\left(\partial_{g}^{j} \mathscr{F}_{e m}^{j}-\partial_{g}^{j} \mathscr{F}_{g}^{j}\right) ; \\ \mathfrak{J}_{(e m-g) /(g-e m)}^{k} & =\left(\partial_{e m}^{0} \mathscr{F}_{g}^{k}+\epsilon_{i j k} \partial_{g}^{i} \mathscr{F}_{e m}^{j}\right) e_{k} ; \\ \mathfrak{J}_{(g-e m) /(e m-g)}^{k} & =\left(-\partial_{g}^{0} \mathscr{F}_{e m}^{k}+\epsilon_{i j k} \partial_{e m}^{i} \mathscr{F}_{g}^{j}\right) e_{k} .\end{cases}
$$

So, the quantum equations for octonionic hot and cold dark matter may easily be expressed in the terms of simpler and compact notation of octonions representations. From the foregoing analysis we find the matter density of octonionic cold dark matter (OCDM) i.e. $\left(0.10 \leq h^{2} \Omega_{C D M} \leq 0.32\right)$ is grater than that of octonionic hot dark matter (OHDM) as $\left(0.00 \leq h^{2} \Omega_{H D M} \leq 0.12\right)$. As such, there exists more cold dark matter than that of hot dark matter in nature supported by octonionic field equations.

\section{References}

[1] L. E. Dickson, "On Quaternions and Their Generalization and the History of the Eight Square Theorem", Ann. Math., 20 (1919), 155.

[2] W. R. Hamilton, "Elements of Quaternions", Chelsea Publications Co., New York, (1969).

[3] P. G. Tait, "An elementary Treatise on Quaternions", Oxford Univ. Press (1875).

[4] A. Cayley, "An Jacobi's elliptic functions, in reply to the Rev. B. Bornwin; and on quaternion", Phil. Mag., $\underline{\mathbf{2 6}}$ (1845), 208.

[5] R. P. Graves, "Life of Sir William Rowan Hamilton", 3 volumes, Arno Press, New York, (1975).

[6] J. C. Baez, "The Octonions", Bull. Amer. Math. Soc., $\underline{39}$ (20R. Bond and A. Szalay, Astrophys. J. 274 (1983) 443.01), 145.

[7] K. Morita, "Quaternionic Variational Formalism for Poincaré Gauge Theory and Supergravity", Prog. Theor. Phys., $\underline{73}$ (1985), 999.

[8] K. Morita, "Gauge Theories over Quaternions and Weinberg-Salam Theory", Prog. Theor. Phys., 65 (1981), 2071.

[9] K. Morita, "Quaternionic Weinberg-Salam Theory", Prog. Theor. Phys., $\underline{67}$ (1982), 1860. 
[10] S. Catto, "Exceptional Projective Geometries and Internal Symmetries", eprint: arXiv: hep-th/0302079 (2003).

[11] S. Catto, "Symmetries in Science:from the rotation group to quantum algebras", Ed. B. Gruber, Plenum Press, $\underline{\mathbf{6}}$ (1993), 129.

[12] R. Foot and G. C. Joshi, "Space-time symmetries of superstring and Jordan Algebras", Int. J. Theor. Phys., 28 (1989), 1449.

[13] R. Foot and G. C. Joshi, "String theories and the Jordan algebras", Phys. Lett., B199 (1987), 203.

[14] T. Kugo and P. Townsend, "Supersymmetry and the Division Algebras", Nucl Phys., B221 (1983), 357.

[15] C. A. Manogue and A. Sudbery, "General solutions of covariant superstring equations of motion", Phys. Rev., D40 (1989), 4073.

[16] J. Schray, "Octonions and Supersymmetry", Ph. D. thesis, Department of Physics, Oregon State University, (1994) (un published).

[17] K. S. Abdel-Khalek Mostafa, "Ring Division Algebra, Self Duality and Supersymmetry", eprint: arXiv: hep-th/0002155 (2000).

[18] M. Günaydin and F. Gürsey, "Quark structure and octonions", J. Math. Phys., $\underline{14}$ (1973), 1651.

[19] K. Imaeda, "Quaternionic formulation of tachyons, superluminal transformations and a complex space-time", Lett. Nuovo Cimento, $\underline{50}$ (1979), 271.

[20] R. Penny, "Octonions and the Dirac equation", Amer. J. Phys., 36 (1968), 871.

[21] A. Gamba, "Peculiarities of the Eight Dimensional Space", J. Math. Phys., $\underline{8}$ (1967), 775 .

[22] B. C. Chanyal, P. S. Bisht and O. P. S. Negi, "Generalized Octonion Electrodynamics", Int. J. Theor. Phys., $\underline{49}$ (2010), 1333.

[23] B. C. Chanyal, P. S. Bisht and O. P. S. Negi, "Generalized Split-Octonion Electrodynamics", Int. J. Theor. Phys., $\underline{50}$ (2011), 1919.

[24] B. C. Chanyal, P. S. Bisht, Tianjun Li and O. P. S. Negi, "Octonion Quantum Chromodynamics", Int. J. Theor. Phys., $\underline{51}$ (2012), 3410.

[25] B. C. Chanyal, P. S. Bisht and O. P. S. Negi, "Octonionic non-Abelian Gauge Theory", Int. J. Theor. Phys., $\underline{\mathbf{5 2}}$ (2013), 3522.

[26] B. C. Chanyal, P. S. Bisht and O. P. S. Negi, "Octonion and conservation laws for dyons", Int. J. Mod. Phys. A, $\underline{\mathbf{2 8}}$ (2013), 1350125. 
[27] B. C. Chanyal, P. S. Bisht and O. P. S. Negi, "Octonion Electrodynamics in Isotropic and Chiral Medium", Int. J. Mod. Phys. A, $\underline{29}$ (2014), 1450008.

[28] B. C. Chanyal, “Octonion massive electrodynamics”, Gen. Relativ. Gravit., $\underline{46}$ (2014), 16461.

[29] B. C. Chanyal, P. S. Bisht and O. P. S. Negi, "Octonion Representation of the Superstring Theory”' Int. J. Eng. Res. \& Tech., $\underline{\mathbf{2}}$ (2013), 1459.

[30] B. C. Chanyal, P. S. Bisht and O. P. S. Negi, "Octonion model of dark matter", J. Theor. Phys., $\underline{\mathbf{2}}$ (2013), 23.

[31] B. C. Chanyal, "Octonion symmetric Dirac-Maxwell equations", Turk. J. Phys., $\underline{\mathbf{3 8}}$ (2014), 174.

[32] M. Günaydin and F. Gursey, "Quark structure and octonions", J. Math. Phys., $\underline{14}$ (1973), 1651.

[33] M. Günaydin and F. Gursey, "Quark statistics and octonions", Phys. Rev. D, $\underline{\mathbf{9}}$ (1974), 3387.

[34] S. Marques and C. G. Oliveira, "Geometrical properties of an internal local octonionic space in curved space time", Phys. Rev. D, $\underline{\mathbf{3 6}}$ (1987), 1716.

[35] Z. Weng, "Octonionic Quantum Interplays of Dark Matter and Ordinary Matter", arXiv: physics,gen-ph/0702019v4, (2008).

[36] Z. Weng, "Octonionic electromagnetic and gravitational interactions and dark matter", arXiv: physics class-ph/0612102 v8, (2009).

[37] B. C. Chanyal, "Role of Octonion in gravity and dark matter", Cliff. Analy. Cliff. Algeb. \& Appl., $\underline{3}$ (2014), 121.

[38] G. Bertone, D. Hooper and J. Silk, "Particle darkmatter: evidence, candidates and constraints", Physics Reports, Vol. $\underline{405}$ (2005), 279.

[39] M. Dine, "Origin of the matter-antimatter asymmetry", Rev. Mod. Phys., $\underline{76}$ (2003), 1.

[40] R. Bond and A. Szalay, "The collisionless damping of density fluctuations in an expanding universe" Astrophys. J. $\underline{274}$ (1983), 443.

[41] K Hagiwara, et al "Review of Particle Physics. Particle Data Group" Phys. Rev. D66 (2002), 010001.

[42] S P Ahlen, et al "Limits on Cold Dark Matter Candidates from an Ultralow Background Germanium Spectrometer", Phys. Lett., B195 (1987), 603.

[43] D S Akerib, et al "New results from the cryogenic dark matter search experiment", Phys. Rev., D68 (2003), 082002. 
[44] M Tegmark, et al "Sources and Detection of Dark Matter and Dark Energy in the Universe", Springer, Berlin (2000), (hep-ph/0008145).

[45] X Wang, et al "Is cosmology consistent?", Phys. Rev., D65 (2002), 123001. 